\title{
Optical Properties of CDS Films by Analysis of Spectral Transmittance
}

\author{
A.J. Aristizábal ${ }^{1}$, M.A. Mikan $^{2}$ \\ ${ }^{1,2}$ Engineering Department, Jorge Tadeo Lozano University, Bogotá, Colombia
}

\begin{abstract}
This paper presents the calculation of the optical properties of thin films of CdS from spectral transmittance measurements using the method of Swanepoel. CdS thin film was grown by evaporation technique which has better advantages in optical and crystalline properties compared to other manufacturing techniques as chemical bath deposition (CBD) and sputtering. The experimental procedure is presented for the transmittance spectrum of CdS thin film through a VIS-IR Oriel spectrophotometer that was automated using virtual instrumentation for data acquisition. After applying the mathematical Swanepoel model, the optical properties of the CdS thin film were obtained: 1499,08nm thick, an absorption coefficient of $2 \times 104 \mathrm{~cm}^{-1}$ for a wavelength of 500nm and an optical gap of $2,38 \mathrm{eV}$. These results indicate that the CdS thin film is suitable for use as optical window in the manufacture of solar cells.
\end{abstract}

Keywords: thin films, optical properties, solar cell, virtual instrumentation.

\section{Introduction}

The Photovoltaic (PV) devices are designed and fabricated mainly to harvest all possible photons, and create a useful electrical power output [1].

Group II-VI semiconductor (CdS, ZnS) thin films have attracted considerable attention from the research community because of their wide use in the fabrication of solar cells and other optoelectronic devices [2,3]. In recent years, extensive research has been carried out on the area of preparation and characterization of $\mathrm{CdS}$ thin films due to its application in different type of most promising photovoltaic solar cells such as CdTe [4], CZTS [5] and CIGS [6].

CdS thin film in a solar cell structure does not just act as a window layer, but contribute to carrier generation due to its high photosensitivity $[7,8]$. Cadmium sulfide (CdS) films have transparency properties in the visible range, acoustic characteristics, high electrochemical stability and excellent electronic properties [9]. It has also been used in electronic and optoelectronic devices [10].

Since 1970s, many techniques, such as chemical bath deposition (CBD) [11,12], sputtering [13], and pulse laser ablation [14], etc. for fabricating CdS thin films have been developed. In CdS-based solar cells, the optical and electrical properties of the CdS films are significant for improving the whole cell device performance $[15,16]$. The higher carrier density and wider band gap for CdS films are the vital factor in improving the open circuit voltage of the solar cell $[15,16]$.

Here we report the optical properties of CdS thin film grown by evaporation technique and the results indicate that it is an appropriate film to be used as optical window in solar cells.

\section{Theoretical Analysis}

The optical constants of the material can be determined using different methods [17]. In this paper the mathematical method developed by Swanepoel [18] will be used to calculate the optical constants of thin films deposited on a transparent substrate from spectral transmittance measurements and theoretical calculations which take into account interference effects [19].

Fig. 1 shows a thin film deposited on a glass substrate.

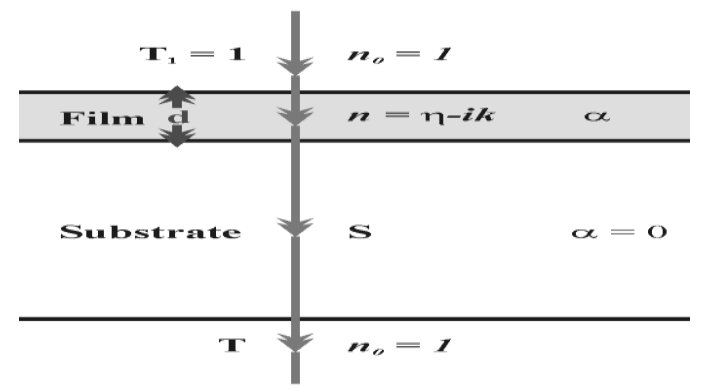

Fig. 1. A semiconductor thin film on a transparent substrate $[18,19]$. 
It is assumed: a film thickness $(\mathrm{d})$, a complex refractive index $(\eta)$ and an extinction coefficient $(\mathrm{k})$ that is expressed in terms of the absorption coefficient $(\alpha)$. The substrate has a thickness of several orders of magnitude greater than that of the film and a refractive index (s).

The typical spectrum of transmittance of a semiconductor thin film is presented in Fig. 2.

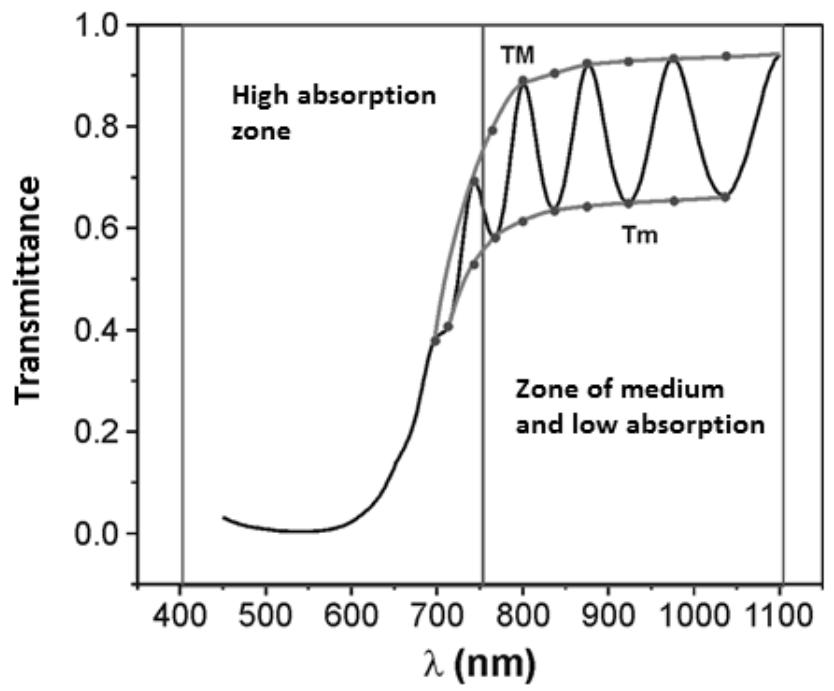

Fig. 2. Typical transmittance spectrum of a semiconductor thin film $[18,19]$.

This method makes a rigorous analysis of interference effects observed in transmittance curves as a result of the superposition of the reflected and transmitted beams at interfaces film/air and substrate/film. This transmittance spectrum has two different areas: the first one is the high absorbency zone where the value of the transmittance drastically decreases, in this area the optical gap of the material is determined; and the second one is the middle absorption zone; which determine the refractive index and we extrapolate the high absorbency zone using Cauchy's equation.

\subsection{Cauchy's equation}

The first approach accepted to represent the dispersion curve normal (n) was made by Cauchy in 1836. This can be written as:

$n=A+\frac{B}{\lambda^{2}}+\frac{C}{\lambda^{4}}$

Where $\mathrm{A}, \mathrm{B}$, and $\mathrm{C}$ are constants of each substance and $\lambda$ is the wavelength in $\mathrm{nm}$.

To find the value of the three constants, it is necessary to know the value of $\mathrm{n}$ for three different wavelengths. For ease of calculation only the first two terms of Cauchy's equation are used, therefore it requires at least two values of $\mathrm{n}$ with their respective wavelengths, to calculate the values of the constants $\mathrm{A}$ and $\mathrm{B}$. Therefore, Cauchy's equation is simplified as:

$n=A+\frac{B}{\lambda^{2}}$

The refractive index $\mathrm{n}$ must meet the following criteria:

- The refractive index increases as the wavelength decreases.

- The rate of increase is larger at short wavelengths.

\subsection{Equation to determine the value of the transmittance}

The expression for determining the value of a transmittance T system as shown in Fig. 2 is $[18,19]$ :

$T=T(\lambda, s, n, d, \alpha)$

However it is convenient to express the equation in terms of $n(\lambda)$ and absorbance $x(\lambda)$, where $x$ is defined below.

$T=T(n, x)$

Rigorous expression for $\mathrm{T}$ transmittance value is the following: 


$$
T=\frac{A x}{B-C x+D x^{2}}
$$

Where:

$$
\begin{gathered}
A=16 s\left(n^{2}+k^{2}\right) \\
B=\left\{(n+1)^{2}+k\right\}\left\{\left\{(n+1) \cdot\left(n+s^{2}\right)+k^{2}\right\}\right. \\
C=2 \cdot\left\{\left(n^{2}-1+k^{2}\right) \cdot\left(n^{2}-s^{2}+k^{2}\right)-2 k^{2}\left(s^{2}+1\right)\right\} \cos \phi \\
-2 \cdot k \cdot\left\{\left(2\left(n^{2}-s^{2}+k^{2}\right)+\left(s^{2}+1\right) \cdot\left(n^{2}-1+k^{2}\right)\right\} \operatorname{sen} \phi\right. \\
D=\left\{(n-1)^{2}+k^{2}\right\}\left\{\left((n-1) \cdot\left(n-s^{2}\right)+k^{2}\right\}\right. \\
\Phi=4 \pi \mathrm{d} / \lambda \\
\mathrm{x}=\exp (-\alpha \mathrm{d}) \\
\alpha=4 \pi \mathrm{k} / \lambda
\end{gathered}
$$

\section{Experimental Procedure}

Fig. 3 shows the flowchart of the process for calculating the optical constants from a transmittance spectrum.

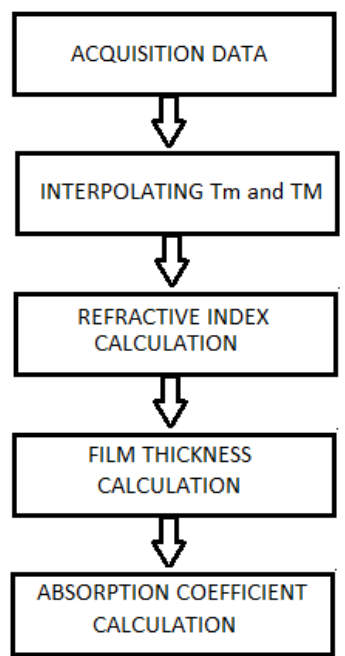

Fig. 3. Flowchart for calculating the optical constants $[19,20]$.

The first step is to perform data acquisition transmittance of the CdS thin film. The spectral transmittance was taken with a VIS-IR Oriel spectrophotometer (see Fig. 4) [19, 20, 21].

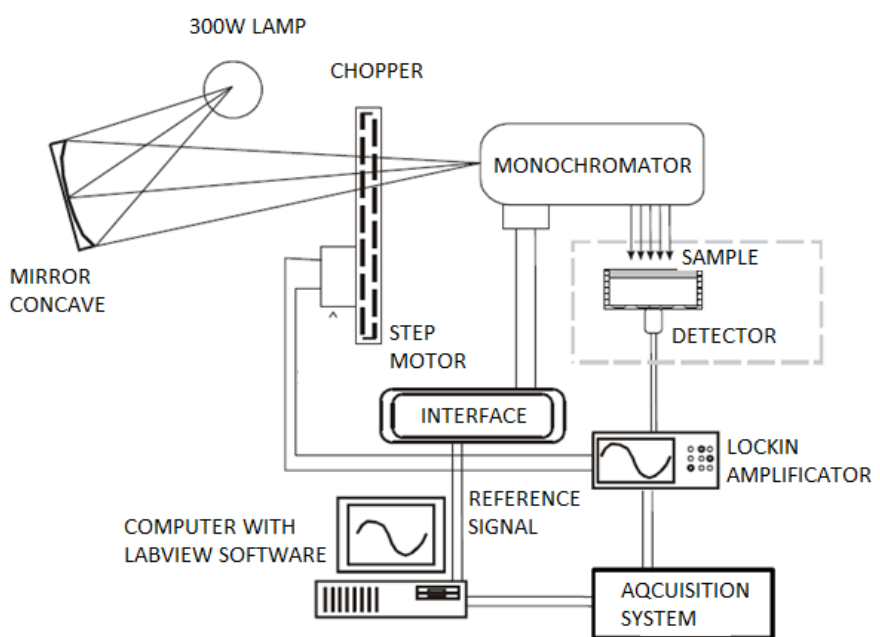

Fig. 5. Block diagram of VIS-IR Oriel spectrophotometer $[19,20]$. 
The following are the technical specifications of the VIS-IR Oriel spectrophotometer:

- $\quad 300 \mathrm{~W}$ halogen lamp: The light focuses on the monochromator via a concave mirror.

- Monochromator: $1 / 4 \mathrm{~m}$ focal distance, usable spectral range $0.3-4 \mu \mathrm{m}$, three diffraction gratings (1200, 600 y $300 \mathrm{~L} / \mathrm{mm}$ ), minimum bandwidth $0.5 \mathrm{~nm}$ (with 1200 grid).

- System phase sensitive detection:

- Optical chopper: converts the light from lamp in light pulses.

- Detector: To measure $\operatorname{JL}(\lambda$ the photocell is used as a detector, to measure Pa a pyroelectric detector is used and to measure transmittance a silicon photodiode is used.

- Lock-in amplifier.

- Electronic computer control system and automatic data acquisition using virtual instrumentation.

Fig. 6 shows the front panel of the virtual instrument developed in LabVIEW software to acquire the spectrum of transmittance.

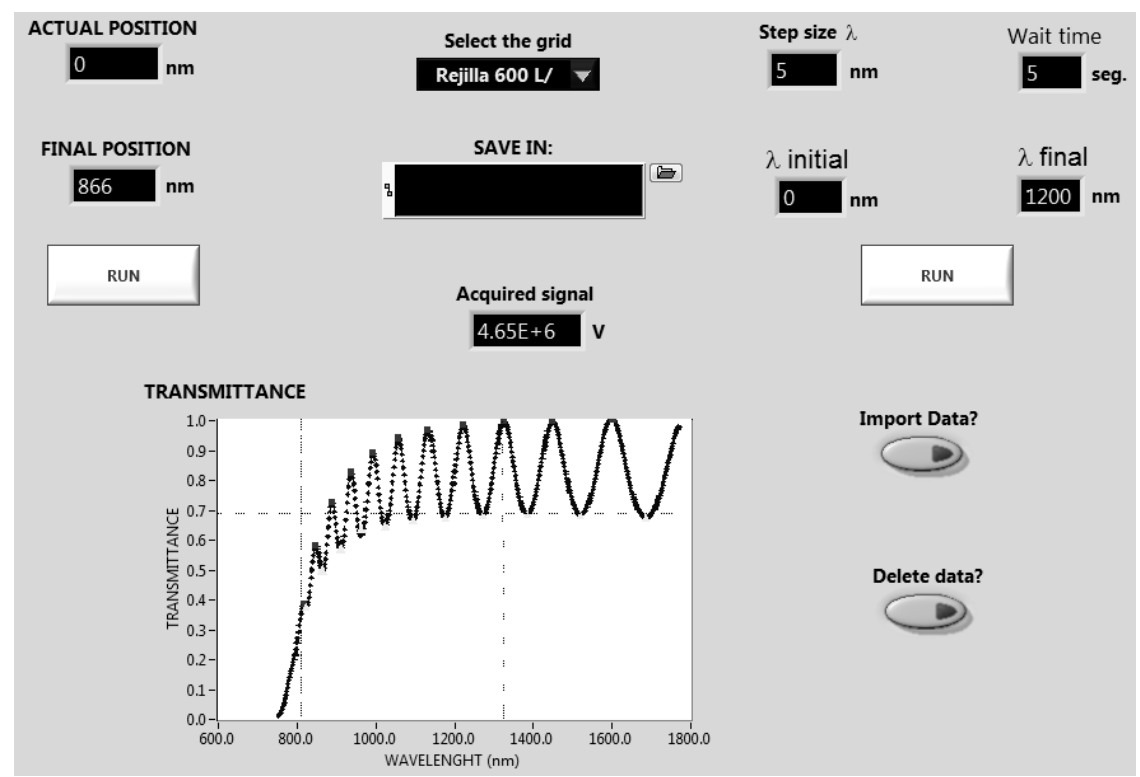

Fig. 6. LabVIEW front panel developed to acquire the transmittance spectrum.

This program uses a LPM-16PnP data acquisition card as hardware and it communicates through the ISA port with the computer.

The virtual instrument developed controls the step motor system that controls the diffraction gratings of the Oriel spectrophotometer that converts white light into monochromatic light and also to measure and process signals through which the spectral transmittance of the thin film $\mathrm{CdS}$ is determined.

Then with the help of a program developed from the procedure described [18,20] treatment transmittance spectrum is performed: For each wavelength where a maximum or minimum interference spectrum is presented, interpolation is performed to calculate the value of transmittance.

Once obtained Tm and TM values, the refractive index is calculated using the procedure developed by Swanepoel $[18,20]$.

For calculating the film thickness a graphical method is used, using as variables the order of interference, the refractive index and the wavelength which it presents the maximum or minimum interference (the order of interference is an integer linked to each interference maximum or half integer if a minimum of interference).

For the calculation of the absorption coefficient equation (13) is used. For this, a numerical method is used as the value of Tcal (k) can't be solved analytically (see equation (5)).

$$
T_{\text {exp }}-T_{\text {cal }}(k) \approx 0, \text { Tolerancia }=10^{-7}
$$

The numerical method used to solve the equation (13) is the Newton-Raphson method, which is shown in Fig. 7. 


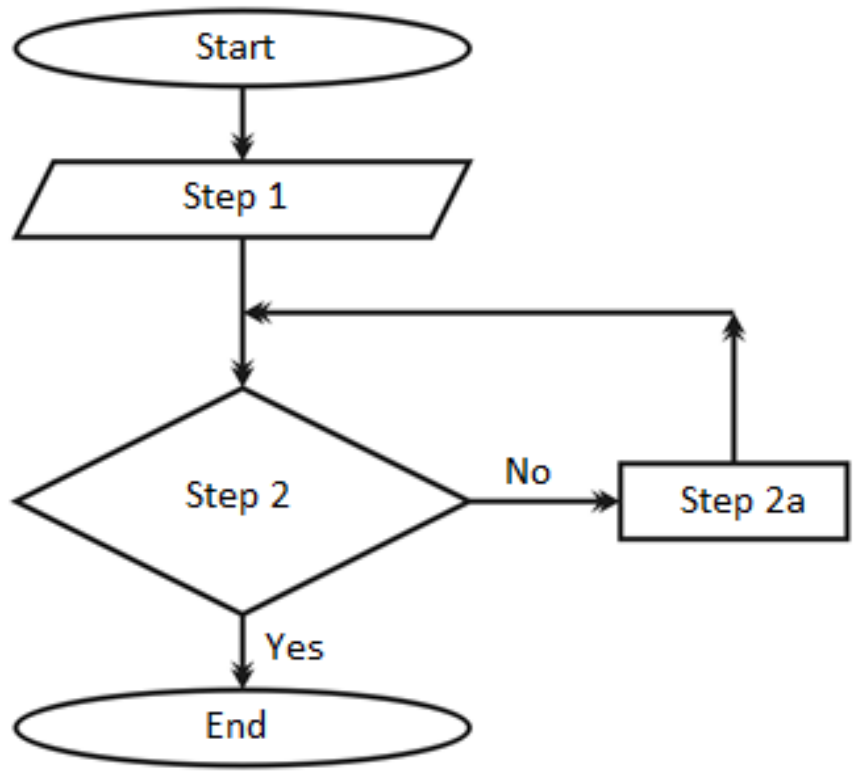

Fig.7. Newton routine for calculating the absorption coefficient $[19,20]$.

The sequence of the method is the following [19,20]:

- Stet 1: in this step the routine starts running with an initial value of $\mathrm{k}$.

- Step 2: the value of the transmittance is calculated and compared with the value of the experimental transmittance, see equation (13). If the difference is not within the allowed tolerance then continue with step $2 \mathrm{a}$, but if the difference is within the allowable tolerance then ends the routine.

- Step 2a: a new value of $k$ is calculated using equation (14) (Newton method).

$$
k_{i}=k_{i-1}-\frac{f\left(k_{i-1}\right)}{\partial f\left(k_{i-1}\right) / \partial k_{i-1}}
$$

This routine is applied to each point of the transmittance's spectrum.

\section{Results and Discussion}

In Fig. 8 the behavior of the CdS thin film transmittance is observed.

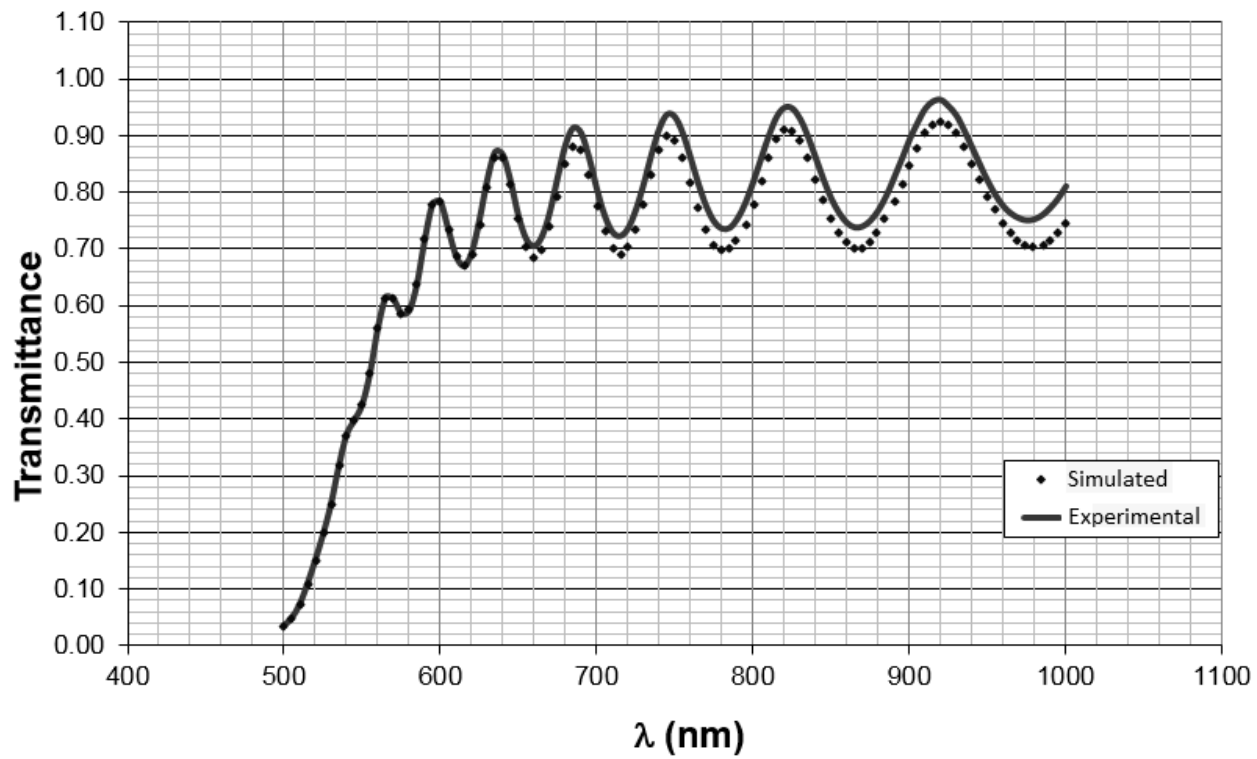

Fig. 8. Transmittance spectrum of CdS thin film. 
It can be seen that the transmittance is present for a wavelength of about $490 \mathrm{~nm}$ onwards; which means that energy values and higher $2.38 \mathrm{eV}$, the incident photons are absorbed by the material and the internal energy increases.

The region of strong transmittance spectrum ranges from $0 \mathrm{~nm}$ to $590 \mathrm{~nm}$, average and weak region from $590 \mathrm{~nm}$ to $780 \mathrm{~nm}$ and the transparent region from $780 \mathrm{~nm}$ to $1050 \mathrm{~nm}$. These values show an error of $0.1 \%$ due to the weak absorption $\alpha$ is very small. In the medium absorption zone $\alpha$ is large and the transmittance decreases.

\subsection{Refractive index (n)}

Fig. 9 shows the variation of the CdS thin film refractive index versus wavelength.

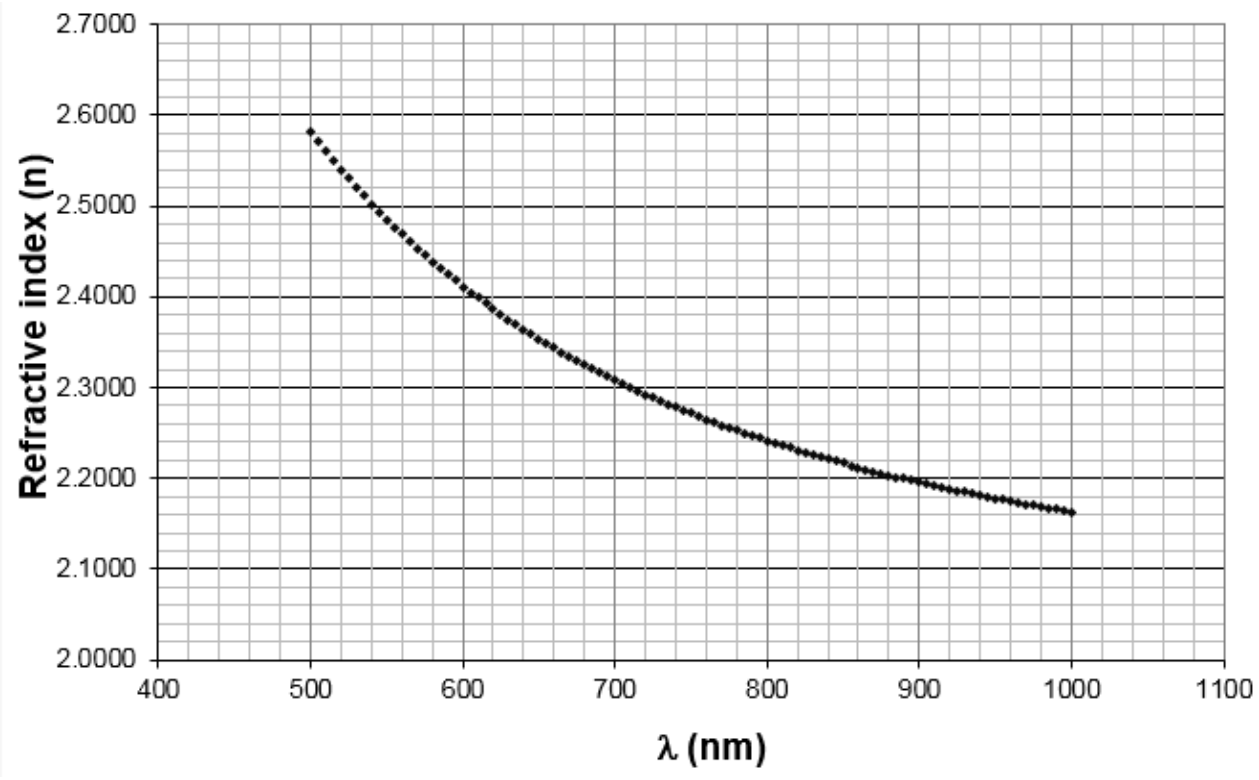

Fig. 9. Variation of refractive index vs. Wavelength.

To calculate the refractive index (n) different values of wavelength $\lambda$ were used and the theoretical value of the constant for the refractive index of the substrate $s=1.51$, in regions of high, medium and weak absorption. The decreasing trend observed in Fig. 9 is due to the increasing refractive index related to the increasing film thickness. The resulting values of $n$, are calculated by the ends of the transmittance spectrum.

\subsection{Thickness (d)}

Fig. 10 shows the relationship $1 \pi$ vs. $n / \lambda$ of $\mathrm{CdS}$ thin film.

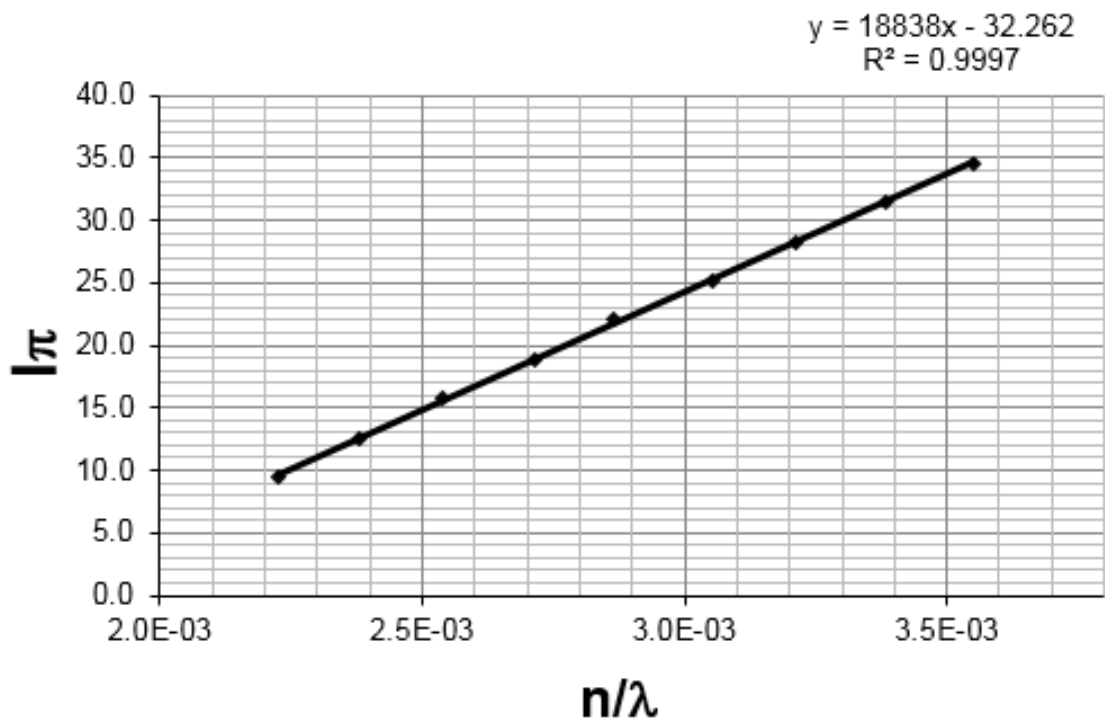

Fig. 10. Variation of $l \pi$ vs. $n / \lambda$ to determine the thickness of the sample of CdS. 
The linear variation of Fig. 10 has a slope of 18838 and a thickness of the film of 1499.0804 nm with close to $1 \%$ error. For calculate it, a graphical method was used using as variables the order of interference, the refractive index and the wavelength which it has a maximum or minimum interference; according to the equation (15) [18]:

$$
2 \mathrm{nd}=\mathrm{m} \lambda
$$

\subsection{Absorption coefficient ( $\square$ )}

In Fig. 11 the variation of the absorption coefficient versus wavelength is shown.

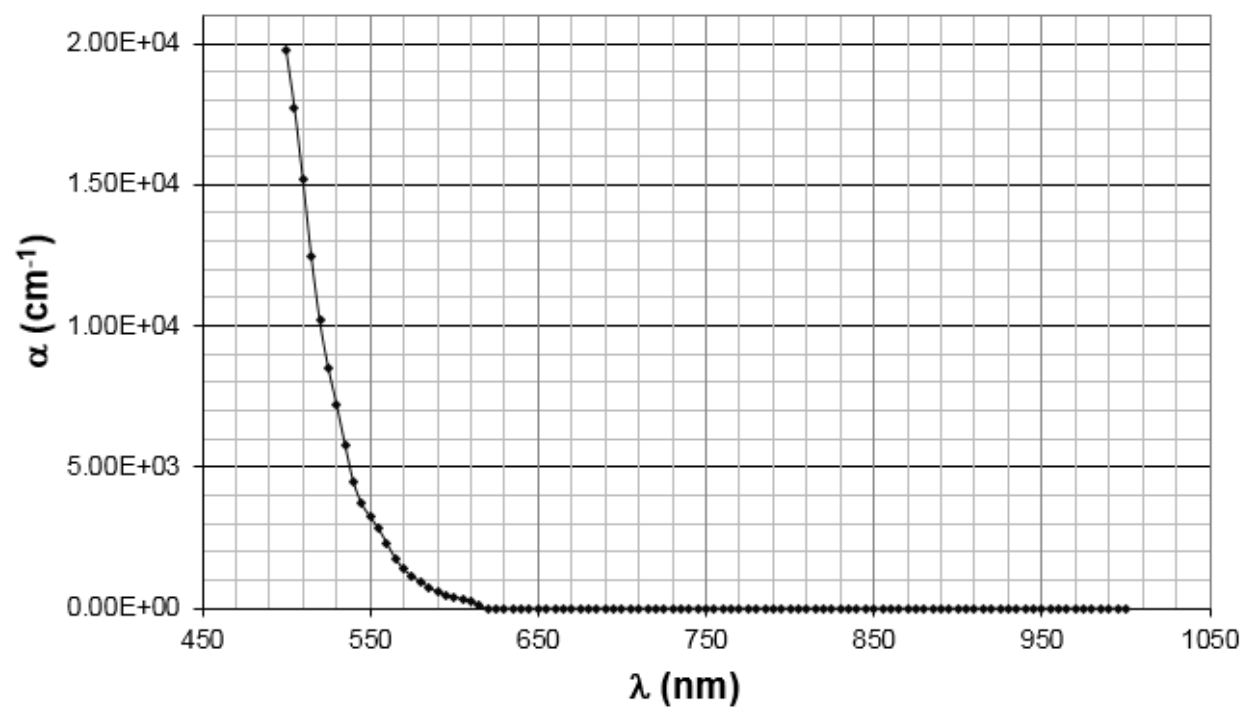

Fig. 11. Variation of the absorption coefficient versus wavelength.

The behavior of the coefficient is proportional to the frequency of the light incident on the thin film The sample absorption capacity increases, but decreases the amount of transmitted light. For wavelengths less than $500 \mathrm{~nm}$ the transmittance becomes zero. In the range of wavelengths from $500 \mathrm{~nm}$ to $1000 \mathrm{~nm}$, it is the medium absorption zone; and the region of low absorption is observable only for lengths greater than $1050 \mathrm{~nm}$.

\subsection{Optical gap} coefficient.

Fig. 12 shows the procedure for calculating the optical gap of the CdS thin film from the absorption

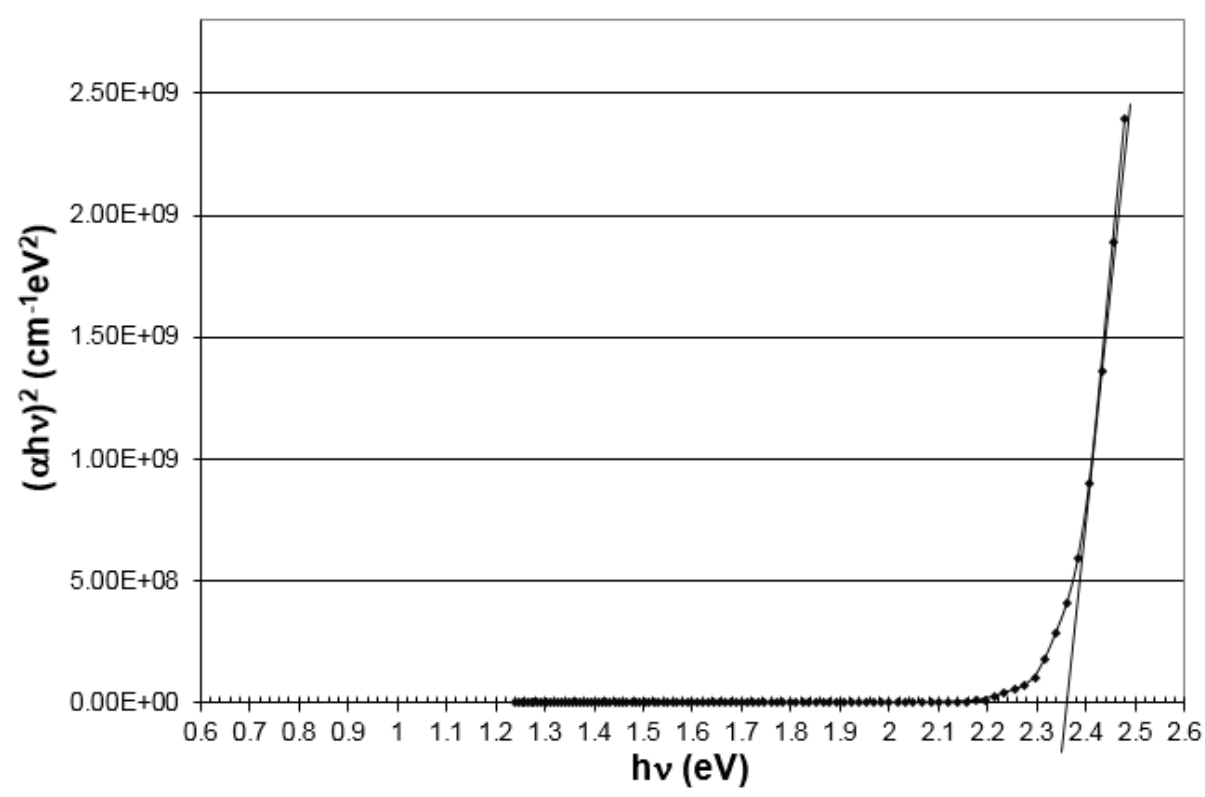

Fig. 12. Curve of the absorption coefficient versus energy to determine the optical gap. 
Given the correlation between the absorption coefficient and photon energy incident of Fig. 12, a straight axis intersecting the energy is drawn. This intersection occurs in the axis eV in $2.38 \mathrm{eV}$ and it represents the value of the optical gap of the CdS film. This means that photons should influence with an energy greater than or equal to $2.38 \mathrm{eV}$ and approximate wavelength of $490 \mathrm{~nm}$ to excite an electron from the valence band and generate the transition to the conduction band of CdS material.

\section{Conclusion}

The Swanepoel method can be considered a suitable method for determining the optical constants that it has a relatively smaller percentage of errors that confirms its high precision. Thanks to the application of virtual instrumentation and Swanepoel method was possible to automate the process of calculating the optical properties of semiconductor thin films.

In this work, we presented the calculation of the optical properties of a thin film that acts as optical window in a solar cell; but the procedure described and the experimental setup can also be applied in thin films responsible for the absorption of photons and the generation of electrons in solar cells. The results indicate that the values of the refractive index gradually grow with increasing film thickness due to variation of density with the thickness, noting the direct relationship between these parameters.

Sample value of $1499.0804 \mathrm{~nm}$ thickness was obtained, a slope of 18838 and the intercept -32.262 The optical gap of the sample was $2.38 \mathrm{eV}$. The region exhibiting greater photon absorption is in a range of $490 \mathrm{~nm}$ to $620 \mathrm{~nm}$. For wavelengths less than $620 \mathrm{~nm}$ the absorption coefficient increases rapidly. The refractive index increases when the incident wavelength on the sample decreases.

The use of LabVIEW software allows us to upgrade the operation of VIS-IR Oriel spectrophotometer with relatively low costs and is a tool that improves hardware systems responsible for the characterization of solar cells as photoconductivity measurements, electrical properties and I-V characteristic.

\section{Acknowledgements}

This work was developed with the support of the Semiconductor Materials \& Solar Energy Group from the Physics Department, National University of Colombia in Bogota. Special thanks to Gerardo Gordillo Guzman and Eduard Ricardo Romero Malagon by the laboratories, equipment and support provided.

\section{References}

[1] A.A. Ojo, I.M. Dharmadasa, 15.3\% efficient graded bandgap solar cells fabricated using electroplated CdS and CdTe thin films, Solar Energy 136 (2016) 10-14.

[2] Y. Gu, E.S. Kwak, J.L. Lensch, J.E. Allen, T.W. Odom, L.J. Lauhon, Near-field scanning photocurrent microscopy of a nanowire photodetector, Appl. Phys. Lett. 87 (2005) 043111-043113.

[3] P.K.C. Pillai, N. Shroff, A.K. Tripathi, A study of the photoconducting propertiesof $\mathrm{CdSSe}(\mathrm{Cu})$ with a view to its use in solid-state image intensifiers, J. Phys., D:Appl. Phys. 16 (1983) 393-399.

[4] N. Amin, K. Sopian, M. Konagai, Numerical modeling of CdS/CdTe andCdS/CdTe/ZnTe solar cells as a function of CdTe thickness, Sol. Energy Mater.Sol. Cells 91 (2007) 1202-1208.

[5] W. Hongxia, Progress in thin film solar cells based on Cu2ZnSnS4, Int. J. Pho-toenergy 2011 (2011) 1-10.

[6] R. Kannan, et al., Properties of 19.2\% efficiency ZnO/CdS/CuInGaSe2thin-filmsolar cells, Prog. Photovolt.: Res. Appl. 11 (4) (2003) 225-230.

[7] L. Chu Ting, S. Chu Shirley, Thin film II-VI photovoltaics, Solid-State Electron.38 (1) (1995) 533-549.

[8] P.K. Nair, J. Campos, M.T.S. Nair, Opto-electronic characteristics of chemicallydeposited cadmium sulphide thin films, Semicond. Sci. Technol. 3 (2) (1988) 134-145.

[9] L. Huang, Z.L. Wei, F.M. Zhang, X.S. Wu, Electronic and optical properties of CdS films deposited by evaporation, Journal of Alloys and Compounds 648 (2015) 591-594.

[10] X.G. Peng, M.C. Schlamp, A.V. Kadavanich, J. Am. Chem. Soc. 119 (1997) 7019-7029.

[11] N.R. Pvaskar, C.A. Menezes, Sinhaaaob, J. Electro Chem. Soc. 124 (1977) 743 - 748.

[12] asn Murthy, K.S. Reddy, J. Power Sources 13 (1984), 59 - 167.

[13] M. Shao, A. Fischer, D. Grecu, Appl. Phys. Lett. 69 (1996) 3045 - 3047.

[14] B. Ullrich, H. Sakai, Y. Segawa, Thin Solid Films 385 (2001) 220 - 224.

[15] Arturo Morales-Acevedo, Sol. Energy Mater. Sol. Cells 90 (2006) 2213 - 2220.

[16] X. Wu, Y. Yan, R.G. Dhere, Y. Zhang, J. Zhou, C. Perkins, B. To, Phys. Stat. sol. 1 (2004) 1062 - 1066.

[17] E. D. Palik (Ed), Handbook of optical constants of solids/Academia Press, Orlando, ch. 5-7, 1997.

[18] R. Swanepoel, J. Phys. E: Sci. Instrum. 16 (1983) 1214.

[19] E. R. Romero, Estudio de propiedades ópticas y estructurales de materiales usados en la fabricación de celdas solares. Tesis doctoral en Química, Universidad Nacional de Colombia, Sede Bogotá, 2008.

[20] M. Gracia, F. Rojas and G. Gordillo. 20th European photovoltaic Solar Energy Conference, Barcelona, España, 2005 p.1874-1877.

[21] C. Calderón, J. Clavijo, E, Romero, G. Gordillo, Síntesis y estudio de propiedades ópticas y estructurales de películas delgadas de $\mathrm{Cu}(\mathrm{In}, \mathrm{Ga}) \mathrm{Se} 2$ y nuevos materiales fotovoltaicos, Revista Colombiana de Física, Vol. 42, No. 3 de 2010. 